\title{
Water Ascends in Woody Plants: So What?
}

\author{
K. Steppe \\ Laboratory of Plant Ecology, Faculty of Bioscience Engineering, Ghent University, \\ Coupure links 653, B-9000 Gent, Belgium \\ kathy.steppe@UGent.be
}

Keywords: dynamic water transport, transpiration, plant sensors, mechanistic plant modelling, early warning, irrigation scheduling, stress detection, speaking plant

\begin{abstract}
Woody plants are often considered as static individuals, taking up water via the root system and losing it again via leaf transpiration. Quite boring one might conclude when considering that more than $95 \%$ of the water taken up by the roots is transpired by the leaves. But the story suddenly becomes much more intriguing when the dynamics happening during the ascent of water in woody plants are included. This keynote talk will elaborate on water transport in woody plants, highlighting where the dynamics come from, what the relevance is and which tissues are involved. The power to decipher water transport dynamics with plant-based measurements, where sap flow and stem diameter variations are at the forefront, will be illustrated. The concept of plant-based measurements will be complemented with a viewpoint on how sophisticated mechanistic water transport models can assist in plant-based irrigation scheduling or early warning and stress detection systems.
\end{abstract}

\section{INTRODUCTION}

It is widely acknowledged by the entire horticultural community that we are in an era where growers need more than green fingers alone to remain competitive on national and international markets. There is an important need for more objective measurements, as well as early warning and stress detection systems. The challenge is to see the invisible by, for instance, applying plant sensors that enable fast detection of stress symptoms, days before visual signs become apparent. Only then we can start doing the impossible and evolve towards true plant-based control systems. This so-called speaking plant approach dates back to the year 1978, when Udink ten Cate and co-workers already outlined the opportunities of direct sensing of plant responses as part of computer-controlled greenhouse management (Udink ten Cate et al., 1978).

To fully comprehend the contribution that plant sensors may bring to these new developments and trends in horticulture, we should stop considering plants as static individuals that only take up water via the root system and lose it again via leaf transpiration. If we want to exploit the full potential of the speaking plant approach, it is necessary to understand the dynamics during the ascent of water in the plants.

\section{WHY WATER TRANSPORT?}

Water transport dynamics are the focus of this keynote paper, because the resulting plant water status is probably the most important factor that has to be controlled if one wants to maximise yield and quality of horticultural products (Baille, 1992; Jones and Tardieu, 1998). Today, measurements of sap flow and stem diameter variation are at the forefront of investigations of the dynamics of water transport in plants. Sap flow is interesting, because it enables quantification of the whole plant water use with minimal 
disruption to the transpiration conditions (Schurr, 1998; Smith and Allen, 1996; Steppe et al., 2010). Stem diameter variations are interesting because they give information on both water transport dynamics and radial stem growth (e.g., Steppe et al., 2006; De Swaef and Steppe, 2010). One of the reasons why sap flow sensors and linear variable displacement transducers (LVDTs) measuring stem diameter variations present a promising future for horticultural application is because they display the integrated response of the plant on changes in its microclimate and soil conditions. If used simultaneously, these two sensors also offer unique opportunities to extent our fundamental knowledge on plant functioning, which is crucial for the development of any successful plant-based control system.

The success of the combined use of the sensors lies in the tight link that exists between sap flow and stem diameter variations (Fig. 1). The dynamic or non-stationary flow conditions emerge from a time lag that exists between water loss by leaf transpiration and water uptake by roots (e.g., Schulze et al. 1985; Steppe et al., 2002). To overcome these temporal imbalances between water loss and uptake, internal water reserves are depleted during the day and subsequently replenished overnight causing the diameter of the stem to shrink and swell accordingly (e.g. Herzog et al. 1995; Zweifel et al. 2001; Steppe \& Lemeur 2004). But the stem diameter variation signal also contains additional information, including radial stem growth, which is superimposed on the daily shrinkage and swelling of the stem. An unambiguous interpretation of these different components of the signal can be achieved by mechanistically modelling the stem diameter variations (Steppe et al., 2006).

\section{MECHANISTIC PLANT MODELLING}

Raw data from the sensors are not useful without a subsequent analysis and interpretation of the data. It is therefore necessary to implement some intelligence in the data processing. This can be best achieved by mechanistic modelling (Steppe et al., 2006). To translate our earlier concept of depletion and replenishment of internal water, as well as radial growth, the plant stem is modelled as two coaxial cylinders separated by a virtual membrane (Fig. 2). Two flow paths are considered: (1) vertical water flow, and (2) radial water flow between the vertical flow and the water storage compartment, representing the elastic living cells of the bark. Vertical water transport in the stem is described by van den Honert's equation relating flow and water potential gradient (van den Honert, 1948). Because this equation only holds for stationary flow conditions (i.e. water uptake equals water loss) but water from storage pools also contributes to the transpiration stream due to the tight hydraulic link between stored and xylem water (Zweifel et al., 2001; Génard et al., 2001; Steppe et al., 2012), the model also foresees radial water flow. This radial water flow into and out of the stem storage pool causes changes in stem water content and, hence, elastic changes in stem diameter. But it should be noted that beside these elastic diameter changes, the tree stem also grows. To distinguish between radial stem growth and elastic shrinkage and swelling of the stem, Lockhart's equation (1965) can be used:

$$
\text { relative growth }=\phi(\text { turgor pressure }-\Gamma) \text {, }
$$

where $\phi$ is the extensibility of cell walls in relation to irreversible dimensional changes (growth of tissue) $\left(\mathrm{MPa}^{-1} \mathrm{~s}^{-1}\right)$ and $\Gamma$ is the critical wall-yielding threshold value which must be exceeded to produce growth in the stem storage compartment. This equation states that cell expansion is driven by turgor pressure (i.e. positive pressure potential), which irreversible deforms the cell wall as the cell compartment expands. The expansion 
requires turgor above a threshold value $(\Gamma)$ before irreversible deformation begins. More details about the mechanistic plant model are given in Steppe et al. (2006).

\section{WHICH TISSUES ARE INVOLVED?}

To investigate which tissues contribute to the transpiration stream during nonstationary flow conditions, we used non-invasive magnetic resonance imaging (MRI) on 2-year-old oak trees (Quercus robur L.) at the Forschungszentrum Jülich (IBG-2, Plant Sciences). Details of the MRI system and the experimental set-up are described in detail in De Schepper et al. (2012). Fig. 3a shows the MRI image of the stem: white represents a high amount of water per pixel and dark grey represents a low amount of water per pixel. Early and late wood vessels contained much more water than the xylem fibres which are almost black. The bright white band with a high amount of water (up to 95\%) represents the cambium and the youngest secondary phloem and xylem. The large water retention observed in this zone is due to osmotically active compounds present in the cells. The amount of water in the remaining bark was rather low (between $20 \%$ and $40 \%$ ).

To quantify the contribution of the different stem tissues to the total water loss in the stem, a mask was defined which divided the stem into three different zones (Fig. 3b). The mask, indicated in orange, was equal to the initial zone with a high amount of water and defines three zones: (1) a zone outside the mask, containing almost the entire bark; (2) the mask itself, containing the cambium, youngest phloem and xylem; and (3) a zone inside the mask, containing the xylem. While the xylem (zone 3) hardly contributed, the bark (zone 1) was losing the highest amount of water (Table 1). These non-conductive phloem cells are indeed less osmotically active compared to zone 2 . Zone 2 with the highest amount of water indeed contributed less (Table 1), because the high concentration of osmotically active compounds in this zone retained water very effectively. These measurements confirm the abovementioned hypothesis that elastic changes in stem diameter are mainly caused by changes in bark water content (De Schepper et al., 2012).

\section{BRINGING FUNDAMENTAL KNOWLEDGE TO PRACTICE}

\section{Plant-based irrigation scheduling}

Based on the fundamental knowledge about plant functioning and dynamic water transport, useful applications for practice can be designed and built. As a first example, a precision irrigation scheduling system as described by Steppe et al. (2008) will be discussed. This plant-based system takes the irrigation needs (when and how much) of the individual plant into account. In contrast to previous studies using plant-based stress sensing as the basis for irrigation scheduling, plant-based measurements of sap flow and stem diameter variation are now fed into a mechanistic plant model, as described above (Fig. 2) in order to continuously predict stem water potential. The amount of required irrigation water is derived from a time integration of the sap flow profile, while the timing of the irrigation is controlled based on a reference value for the predicted stem water potential. To make this system work, a dynamic model calibration was necessary using a moving window of four days of past data of stem diameter variations. The method was successfully validated with direct measurements of stem water potential and was able to capture the diurnal fluctuations in stem water potential with high temporal resolution. Although the new methodology was successfully tested in a pilot-scale setup with young potted apple trees (Malus domestica Borkh) (Steppe et al., 2008), a shortcoming in the approach is the definition of the static critical reference value, beyond which irrigation is 
necessary. The reference value has been derived from the relationship between midday values of maximum photosynthesis rates and stem water potential (De Swaef et al., 2009), but the real challenge is to have a dynamic reference that changes with environmental conditions and/or plant age. This may be achieved by mechanistic modelling, as demonstrated for leaf temperature measurements by Vermeulen et al. (2012).

\section{Early-warning and stress detection}

Defining dynamic references to which actual measurements can be compared is also crucial for the development of early-warning and stress detection systems. Controlled stress experiments, of which a typical example is displayed in Fig. 4, show that measured stem diameters deviate from the expected pattern (control plant) as soon as stress (drought stress in this particular case) is imposed. This illustrates that we have tools (plant sensors) at hand to see the invisible: stress was indeed detected eight days before visual symptoms (first signs of leaf wilting) became apparent. Again, a proper definition of the critical reference value is needed, beyond which a management action is required. The challenge is to make these reference values dynamic by, for instance, relying on mathematical modelling as advocated by Vermeulen et al. (2012).

\section{CONCLUSIONS}

It is clear that there are new developments and trends in horticulture. Plant sensors and mechanistic models, and our fundamental understanding of water transport dynamics, can make a substantial contribution to these new developments. This becomes feasible due to the significant advances in the field of sensor technology and mechanistic modelling. With adequate measurements, data processing and modelling, the right decisions can be deduced and applied, contributing to the development of the sustainable and competitive greenhouse horticulture of the future.

\section{ACKNOWLEDGEMENTS}

The author is grateful to the sponsors of various aspects of the work presented, including the FWO and the IWT.

\section{Literature Cited}

Baille, A. 1992. Water status monitoring in greenhouse crops. Acta Hort. 304: 15-27.

De Schepper, V., van Dusschoten, D., Copini, P., Jahnke, S., Steppe, K. 2012. MRI links stem water content to stem diameter variations in transpiring trees. J. Exp. Bot. 63: 2645-2653.

De Swaef, T. and Steppe, K. 2010. Linking stem diameter variations to sap flow, turgor and water potential in tomato. Funct. Plant Biol. 37: 429-438.

De Swaef, T., Steppe, K. and Lemeur, R. 2009. Determining reference values for stem water potential and maximum daily trunk shrinkage in young apple trees based on plant responses to water deficit. Agr. Water Man. 96: 541-550.

Génard, M., Fishman, S., Vercambre, G., Huguet, J.-G., Bussi, C., Besset, J. and Habib, R. 2001. A biophysical analysis of stem and root diameter variations in woody plants. Plant Physiol. 126: 188-202.

Herzog, K.M., Häsler, R. and Thum, R. 1995. Diurnal changes in the radius of a subalpine Norway spruce stem: their relation to the sap flow and their use to estimate transpiration. Trees 10: 94-101. 
Jones, H.G. and Tardieu, F. 1998. Modelling water relations of horticultural crops: a review. Sci. Hortic. 74: 21-46.

Lockhart, J.A. 1965. An analysis of irreversible plant cell elongation. J. Theor. Biol. 8: 264-275.

Schulze, E.-D., Cermak, J., Matyssek, R., Penka, M., Zimmermann, R., Vasicek, F., Gries, W. and Kucera, J. 1985. Canopy transpiration and water fluxes in the xylem of the trunk of Larix and Picea trees - a comparison of xylem flow, porometer and cuvette measurements. Oecologia 66: 475-483.

Schurr, U. 1998. Xylem sap sampling - new approaches to an old topic. Trends Plant Sci. 3: 293-298.

Smith, D.M. and Allen, S.J. 1996. Measurement of sap flow in plant stems. J. Exp. Bot. 47: $1833-1844$.

Steppe, K., Cochard, H., Lacointe, A., Améglio, T. 2012. Could rapid diameter changes be facilitated by a variable hydraulic conductance? Plant Cell Envir. 35: 150-157.

Steppe, K., De Pauw, D.J.W., Doody, T.M. and Teskey, R.O. 2010. A comparison of sap flux density using thermal dissipation, heat pulse velocity and heat field deformation methods. Agr. For. Met. 150: 1046-1056.

Steppe, K., De Pauw, D.J.W. and Lemeur, R. 2008. A step towards new irrigation scheduling strategies using plant-based measurements and mathematical modelling. Irrig. Sci. 26: 505-517.

Steppe, K., De Pauw, D.J.W., Lemeur, R. and Vanrolleghem, P.A. 2006. A mathematical model linking tree sap flow dynamics to daily stem diameter fluctuations and radial stem growth. Tree Physiol. 26: 257-273.

Steppe, K., and Lemeur R. 2004. An experimental system for analysis of the dynamic sap-flow characteristics in young trees: results of a beech tree. Funct. Plant Biol. 31: 83-92.

Steppe, K., Lemeur, R. and Samson R. 2002. Sap flow dynamics of a beech tree during the solar eclipse of 11 August 1999. Agr. For. Met. 112: 139-149.

Udink ten Cate, A.J., Bot, C.P.A. and Van Dixtorn, J.J. 1978. Computer control of greenhouse climates. Acta Hort. 87: 265-272.

van den Honert, T.H. 1948. Water transport in plants as a caternary process. Discuss. Faraday Soc. 3: 146-153.

Vermeulen, K., Aerts, J.M., Dekock, J., Bleyaert, P., Berckmans, D. and Steppe, K. 2012. Automated leaf temperature monitoring of glasshouse tomato plants by using a leaf energy balance model. Comput. Electron. Agric. 87: 19-31.

Zweifel, R., Item, H. and Hasler R. 2001. Link between diurnal stem radius changes and tree water relations. Tree Physiol. 21: 869-877. 


\section{Tables}

Table 1. Average contribution of three different stem zones to the total water loss in the stem. The stem zones were defined as depicted in Fig. 3B; here, the main tissues of the different zones are stated in parentheses (adapted from De Schepper et al., 2012)

\begin{tabular}{ll}
\hline Stem zone & $\begin{array}{l}\text { Average water loss of the zone relative } \\
\text { to the total water loss in the stem }(\%)\end{array}$ \\
\hline Zone 1 (bark) & 58 \\
Zone 2 (cambium) & 27 \\
Zone 3 (xylem) & 15 \\
\hline
\end{tabular}




\section{Figures}

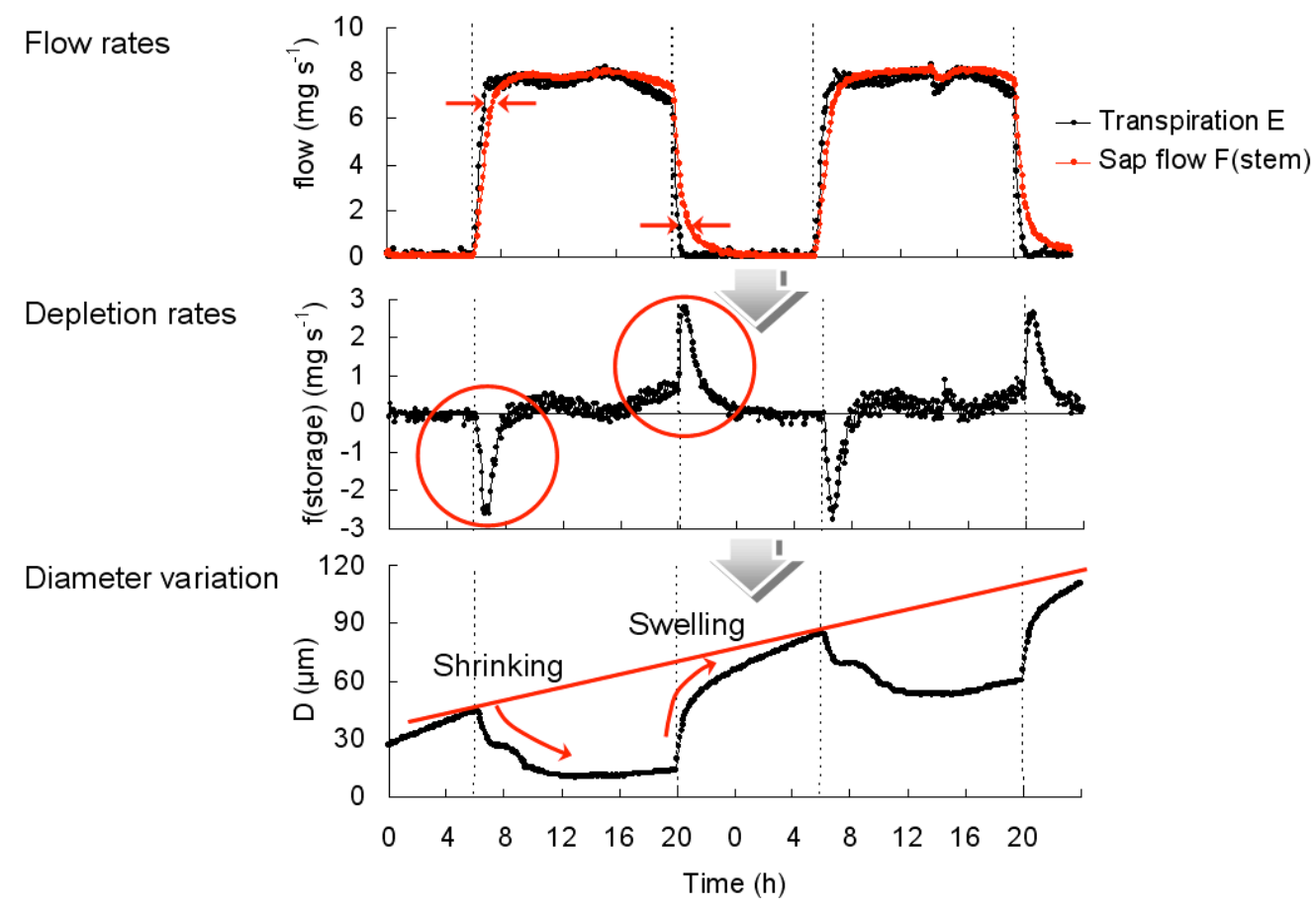

Fig. 1. Tight coupling between sap flow and stem diameter variations, illustrating that the dynamic water flow emerges from a time lag between water loss by leaf transpiration and water uptake by roots. To overcome temporal imbalances, internal water reserves are depleted during the day and subsequently replenished overnight, causing the diameter of the stem to shrink and swell accordingly. The radial stem growth is superimposed on the daily shrinkage and swelling signal of the stem.

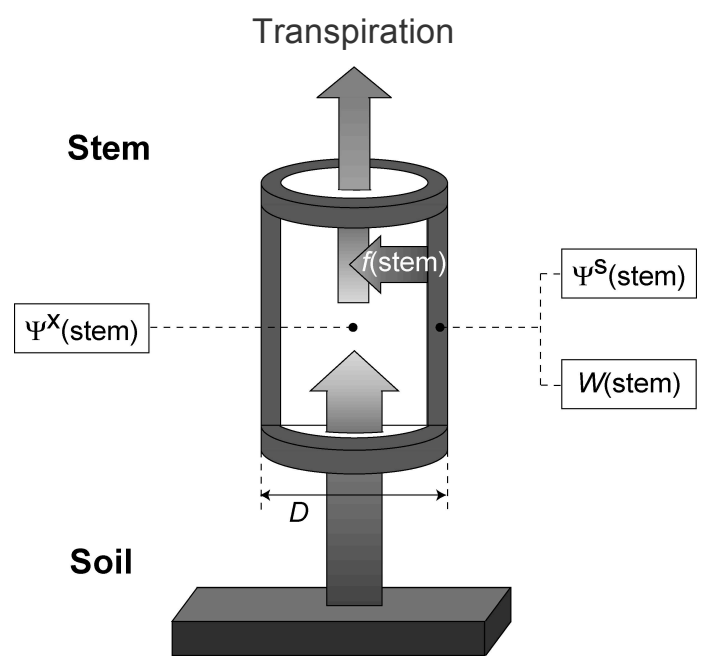

Fig. 2. Schematic representation of the mechanistic plant model. The model is driven by transpiration. The radial flow rate into and out of the stem storage compartment is represented by $f($ stem $)$. Abbreviations: $\Psi^{\mathrm{x}}($ stem $)=$ water potential in the stem xylem compartment; $\Psi^{\mathrm{s}}($ stem $)=$ water potential in the stem storage compartment; $W($ stem $)=$ water content of the stem storage pool; and $D=$ stem diameter. 

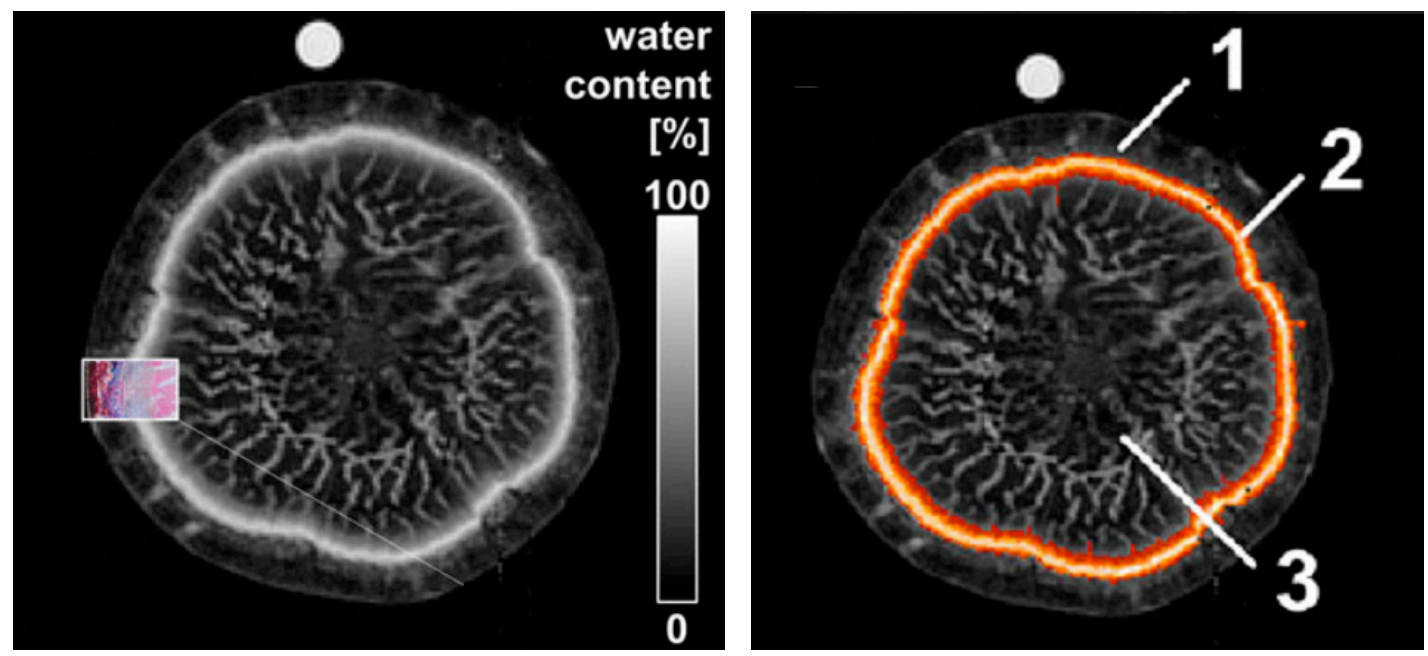

Fig. 3. The amount of water in a stem cross-section of an oak tree measured by MRI (left). The orange mask defines the zone with the high amount of water (white band in left image). Based on this mask three distinct zones are defined: (1) zone 1 is located outside the mask, (2) zone 2 is the mask itself (orange band), and (3) zone 3 is located inside the mask (right) (adapted from De Schepper et al., 2012). Changes in water content of these zones are shown in Table 1.

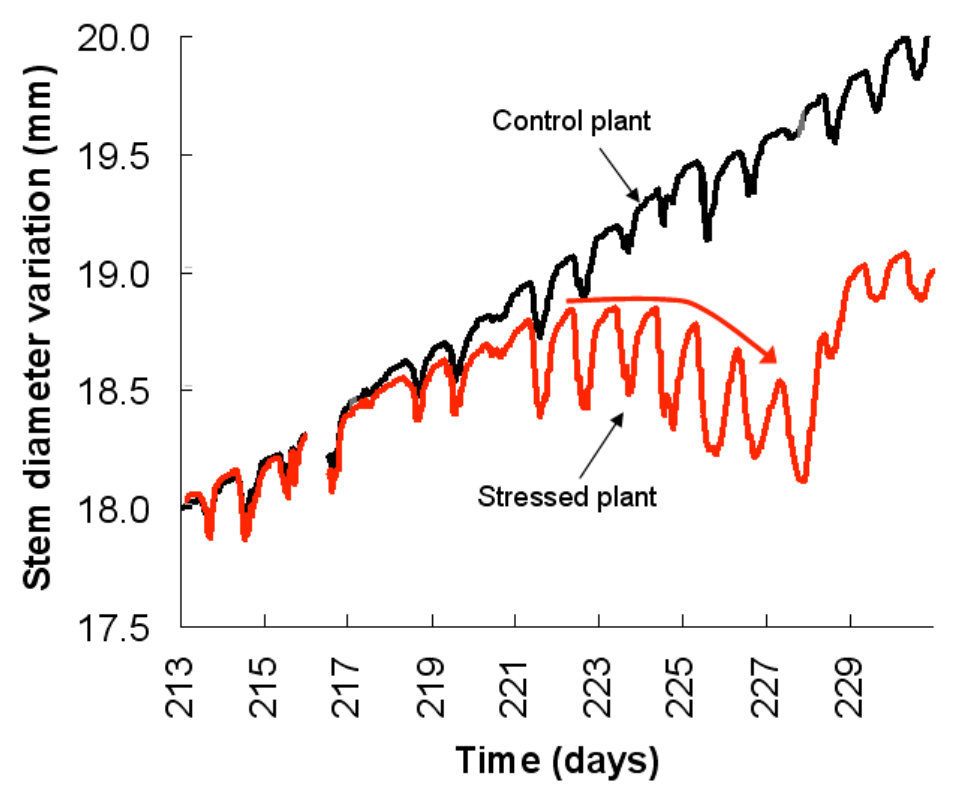

Fig. 4. Continuous pattern of stem diameter variations from LVDT. Data were collected during a drought stress experiment conducted on the apple cultivar 'Mutsu'. The experiment includes a control and a stressed tree. The vertical bars mark the period of drought for the stressed plant. 